\title{
Unsteady free-surface waves due to a submerged body moving in a viscous fluid
}

\author{
Dong-Qiang $\mathrm{Lu}$ \\ Shanghai Institute of Applied Mathematics and Mechanics, Shanghai University, Yanchang Road, Shanghai 200072, China \\ Allen T. Chwang* \\ Department of Mechanical Engineering, The University of Hong Kong, Pokfulam Road, Hong Kong, China
}

(Received 28 August 2004; published 9 June 2005)

\begin{abstract}
Unsteady viscous free-surface waves generated by a three-dimensional submerged body moving in an incompressible fluid of infinite depth are investigated analytically. It is assumed that the body experiences a Heaviside step change in velocity at the initial instant. Two categories of the velocity change, (i) from zero to a constant and (ii) from a constant to zero, will be analyzed. The flow is assumed to be laminar and the submerged body is mathematically represented by an Oseenlet. The Green functions for the unbounded unsteady Oseen flows are derived. The solutions in closed integral form for the wave profiles are given. By employing Lighthill's two-stage scheme, the asymptotic representations of free-surface waves in the far wake for large Reynolds numbers are derived. It is shown that the effects of viscosity and submergence depth on the free-surface wave profiles are respectively expressed by the exponential decay factors. Furthermore, the unsteady wave system due to the suddenly starting body consists of two families of steady-state waves and two families of nonstationary waves, which are confined within a finite region. As time increases, the waves move away from the body and the finite region extends to an infinite V-shaped region. It is found that the nonstationary waves are the transient response to the suddenly started motion of the body. The waves due to a suddenly stopping body consist of a transient component only, which vanish as time approaches infinity.
\end{abstract}

DOI: 10.1103/PhysRevE.71.066303

PACS number(s): 47.15.Gf

\section{INTRODUCTION}

The generation of waves due to a moving body on or below the free surface has been investigated by many researchers in view of its practical importance and theoretical interest. The general mathematical problem posed in terms of the well-known Navier-Stokes equations is extremely difficult to solve. Even if the effect of nonlinear convective terms can be neglected, to solve the resultant linearized equations is still a formidable task. Of the few analytical approaches available for solving linear problems, one is the singularity method, which originates from the method of Green functions for linear partial differential equations, as discussed by Chwang and $\mathrm{Wu}[1]$, Clauser [2], and Dabros [3]. By the superposition principle for linear systems, a body of arbitrary shape can be simulated, at least theoretically, by a discrete or continuous distribution of fundamental singularities while the flow field can be represented by the corresponding distribution of fundamental solutions. The type and distribution of fundamental singularities to be used depend both on the geometry of the moving body and on the nature of the flow. Therefore, the mathematical formulation for a body-induced flow may be characterized by a fundamental singularity, and it is essential to seek the corresponding fundamental solution as the first step to solve the full problem. Based on the potential theories, analytical solutions for steady free-surface waves generated by moving singularities in inviscid fluids are well developed $[4,5]$. The mathematical formulation for

\footnotetext{
*Corresponding author. Fax: (+852) 2858 5415; electronic address: atchwang@hkucc.hku.hk
}

the unsteady free-surface waves generated by a simple source suddenly brought into existence at time $t=0$ and moving with a constant velocity in an inviscid fluid was given by Wehansen and Laitone [4] and the corresponding asymptotic expression for transient free-surface waves was provided by Liu and Tao [6]. The asymptotic expression for transient free-surface waves due to a simple source suddenly stopped at time $t=0$ in an inviscid fluid was provided by Lu and Chwang [7]. It can be seen from Liu and Tao's solution and Lu and Chwang's solution that the transient inviscid waves do not tend to zero as time goes to infinity, which is incompatible with the physical reality. In addition, the effect of the laminar far wake on the free-surface waves for a body moving in a viscous fluid has not been considered by the potential theories.

It is well known that all fluids are naturally viscous. To obtain a better description of free-surface waves in a real fluid, Wu and Messick [8] considered the effect of viscosity on ship waves for two-dimensional cases. Based on an Oseen-type approximation, Cumberbatch [9] studied the effect of viscosity on three-dimensional steady ship waves. Lurye [10] developed an integral formulation for the interaction of steady free-surface waves with viscous wakes. Brard [11] studied the effect of wake and vorticity on the ship waves. Based on the Oseenlet solution, Dugan [12] and Ammicht [13] analytically studied the drag and lift exerted on a submerged plate moving near the free-surface of a viscous fluid without and with the surface tension respectively. Lurye [14] and Chan and Chwang [15,16] obtained analytical solutions for the three-dimensional steady viscous waves due to various Oseenlets and Oseen doublets. Recently, Liu and Tao [17] and $\mathrm{Lu}$ [18] analytically studied the free-surface waves and far wakes generated by a floating body in a viscous fluid 
of infinite depth, in which the floating body is modeled as a surface pressure point. Starting from Lurye's formulation, Lu [19] also reconsidered the steady wave-wake interaction problem in a viscous fluid of infinite depth and provided the full asymptotic expansions of wave profiles in the far wake for large Reynolds numbers, from which the viscous correction for the wave profile is explicitly seen. The validity of the

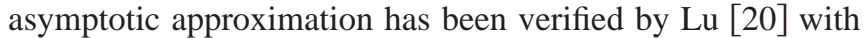
a numerical analysis. The investigation on viscous phenomena has gradually become an expanding branch in the field of free surface flows, as stated by Tyvand [21]. The aforementioned works were based on the assumption that all motions attain a steady state. However, in many situations the body may experience an unsteady motion. Based on the unsteady Stokes equations, Maxey and Riley [22] obtained an analytical solution for fluid motion resulting from a sphere starting from rest and having an impulsive velocity $[\delta(t), 0,0]$, where $\delta()$ is the Dirac delta function. Pozrikidis [23] derived an explicit expression for the three-dimensional oscillatory Stokeslet. It is believed that the convolution-integral formulation of a transient Oseenlet given by Price and Tan [24] and a series of generalized unsteady Oseenlets derived by Chan and Chwang [25] and Shu and Chwang [26] are useful to study the unsteady flow fields associated with a body maneuvering in an unbounded viscous fluid. For the flow in a bounded fluid, Shu and Chwang [27] considered the shorttime slamming effect on the surface waves of a viscous fluid. Based on the formulation and methodology developed by Shu and Chwang [28] for transient Marangoni waves due to the impulsive motion of a submerged singularity, Lu and Chwang [29] derived the wave front and wave profiles generated by a surface-piercing singularity in three dimensions. On the other hand, Liu and Tao [6] studied the viscous freesurface waves due to a suddenly starting Oseenlet and found a finite region of validity for the steady-state solution. Lu and Chwang [30] investigated the two-dimensional free-surface waves due to an oscillatory Oseenlet moving in an incompressible viscous fluid of infinite depth. However, analytical solutions for the transient waves in a viscous fluid have not been obtained and the interaction of a laminar far wake with unsteady free-surface waves has not been fully explored, which are of interest in the present paper.

In this paper, we analytically study the unsteady freesurface waves generated by a point force moving in an incompressible viscous fluid of infinite depth. The point force, mathematically represented by an Oseenlet, is assumed to experience a Heaviside step change in velocity at the initial instant. The general mathematical model for this wave-wake interaction problem is formulated in Sec. II. The analytical solutions for far-field waves due to a suddenly starting and stopping Oseenlet are obtained in Secs. III and IV, respectively. The physical characteristics of the unsteady viscous waves are explicitly discussed in Sec. V. Finally, conclusions are made in Sec. VI.

\section{GENERAL MATHEMATICAL FORMULATION}

The disturbed flow field and the free-surface waves due to a point force moving along a straight line in a stationary

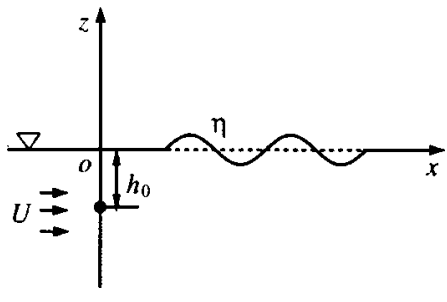

FIG. 1. Definition of the coordinate system.

viscous incompressible fluid with a free surface shall be considered. It is assumed that the point force experiences a Heaviside step change in velocity. Cartesian coordinates are taken fixed on the body, as shown in Fig. 1. The $x$-axis is along the straight path of the moving point while the $z$-axis points vertically upward. Thus the fluid is moving at a uniform velocity $U \mathbf{e}_{x}$, where $U$ is a constant and $\mathbf{e}_{x}$ the unit vector in the $x$ direction. The disturbed flow is assumed to be laminar.

We choose $\rho U^{2}$ as a reference pressure, $U^{2} / g$ as a characteristic length, where $\rho$ is the density of the fluid and $g$ the gravitational acceleration. For simplicity, the velocity is nondimensionalized by $U$, the pressure by $\rho U^{2}$, the distance by $U^{2} / g$, the external force by $\rho U^{2}\left(U^{2} / g\right)^{2}$, and the time by $U / g$. The governing equations are the dimensionless continuity equation

$$
\boldsymbol{\nabla} \cdot \mathbf{u}=0
$$

and the unsteady Oseen equations with a singular force term

$$
\frac{\partial \mathbf{u}}{\partial t}+\frac{\partial \mathbf{u}}{\partial x}=-\nabla P+\varepsilon \nabla^{2} \mathbf{u}+\mathbf{F} \delta\left(\mathbf{x}-\mathbf{x}_{0}\right) H(t)
$$

where $\mathbf{u}=(u, v, w)$ is the disturbed velocity, $P$ the hydrodynamic pressure, which is equal to the total pressure $p$ minus the hydrostatic pressure due to gravity. $\varepsilon=\mu g / \rho U^{3}$, where $\mu$ is the viscosity of the fluid. $\mathbf{F}$ is the singular force located at position $\mathbf{x}_{0}=\left(0,0,-h_{0}\right)$, where $h_{0}$ is the submergence depth. $H()$ is the Heaviside step function. The dimensionless parameter $\varepsilon$ can be regarded as the reciprocal of the Reynolds number with respect to the characteristic length.

For small-amplitude waves, we impose the linearized boundary conditions at the undisturbed free surface $z=0$,

$$
\begin{gathered}
\frac{\partial \eta}{\partial t}+\frac{\partial \eta}{\partial x}=w \\
\varepsilon\left(\frac{\partial u}{\partial z}+\frac{\partial w}{\partial x}\right)=0 \\
\varepsilon\left(\frac{\partial v}{\partial z}+\frac{\partial w}{\partial y}\right)=0 \\
p-2 \varepsilon \frac{\partial w}{\partial z}=0
\end{gathered}
$$

where $p=P-\eta$ is the total pressure on the undisturbed free surface $z=0, \eta$ is the dimensionless elevation of the free surface. 
When the velocity of the point force changes from zero to a constant at the instant $t=0$, the initial velocity and freesurface wave elevation are taken to be those of the quiescent fluid case. When the velocity of the point force changes from a constant to zero, the initial values are taken to be those of the corresponding steady-state solutions.

\section{UNSTEADY VISCOUS FREE-SURFACE WAVES DUE TO A SUDDENLY STARTING OSEENLET}

To simulate the free-surface flow past a moving submerged body, we investigate the theoretical solution for a free-surface flow due to a forcelet $F_{x} \mathbf{e}_{x}+F_{z} \mathbf{e}_{z}$ located at $\mathbf{x}_{0}$, where $F_{x}$ is the strength of the applied drag and $F_{z}$ the strength of the applied lift. Since the problem considered here is an initial-boundary-value one with a singularity, we may regard the disturbed flow $(\mathbf{u}, P)$ as the sum of an unbounded singular Oseen flow $\left(\mathbf{u}_{S}, P_{S}\right)$, which represents the effect of viscous wakes, and an Oseen flow $\left(\mathbf{u}_{R}, P_{R}\right)$, which represents the influence of the free surface and is regular in the whole region. Thus,

$$
\begin{aligned}
& \mathbf{u}=\mathbf{u}_{S}\left(\mathbf{x}, t ; \mathbf{x}_{0}\right)+\mathbf{u}_{R}(\mathbf{x}, t), \\
& P=P_{S}\left(\mathbf{x}, t ; \mathbf{x}_{0}\right)+P_{R}(\mathbf{x}, t) .
\end{aligned}
$$

By taking the Laplace transform over the unsteady Oseen equations with a singular forcing term $\mathbf{F} \delta\left(\mathbf{x}-\mathbf{x}_{0}\right) \delta(t)$, the corresponding Green function can be obtained by means of a straightforward manipulation. The solution of Eqs. (1) and (2) with initial conditions for a quiescent fluid in an unbounded field can be constructed as [31]

$$
\mathbf{u}_{S}\left(\mathbf{x}, t ; \mathbf{x}_{0}\right)=-\frac{1}{4 \pi} \mathbf{F} \cdot\left(\mathbf{I} \nabla^{2}-\boldsymbol{\nabla} \boldsymbol{\nabla}\right) \int_{0}^{t} \frac{1}{r_{t}} \operatorname{erf}\left(\frac{r_{t}}{2 \sqrt{\varepsilon(t-\tau)}}\right) \mathrm{d} \tau,
$$

where $r_{t}=\left\|\mathbf{x}-\mathbf{x}_{0}-t \mathbf{e}_{x}\right\|$,

$$
P_{S}\left(\mathbf{x}, t ; \mathbf{x}_{0}\right)=-\frac{1}{4 \pi} \mathbf{F} \cdot \boldsymbol{\nabla}\left(\frac{1}{r}\right) H(t) .
$$

Furthermore, we write $\mathbf{u}_{R}=\boldsymbol{\nabla} \Phi+\mathbf{V}_{T}$, where $\Phi(\mathbf{x}, t)$ is a scalar potential function and $\mathbf{V}_{T}(\mathbf{x}, t)$ is a solenoidal vector. Therefore, we express the boundary conditions in terms of $\Phi, \mathbf{V}_{T}, \mathbf{u}_{S}, P_{S}$ at $z=0$ as

$$
\begin{gathered}
\frac{\partial \eta}{\partial t}+\frac{\partial \eta}{\partial x}-\left(\frac{\partial \Phi}{\partial z}+w_{T}\right)=w_{S}, \\
2 \frac{\partial^{2} \Phi}{\partial x \partial z}+\frac{\partial u_{T}}{\partial z}+\frac{\partial w_{T}}{\partial x}=-\left(\frac{\partial u_{S}}{\partial z}+\frac{\partial w_{S}}{\partial x}\right), \\
2 \frac{\partial^{2} \Phi}{\partial y \partial z}+\frac{\partial v_{T}}{\partial z}+\frac{\partial w_{T}}{\partial y}=-\left(\frac{\partial v_{S}}{\partial z}+\frac{\partial w_{S}}{\partial y}\right), \\
\frac{\partial \Phi}{\partial t}+\frac{\partial \Phi}{\partial x}+\eta+2 \varepsilon\left(\frac{\partial^{2} \Phi}{\partial z^{2}}+\frac{\partial w_{T}}{\partial z}\right)=P_{S}-2 \varepsilon \frac{\partial w_{S}}{\partial z},
\end{gathered}
$$

where $\left(u_{T}, v_{T}, w_{T}\right)$ and $\left(u_{S}, v_{S}, w_{S}\right)$ are the components of $\mathbf{V}_{T}$ and $\mathbf{u}_{S}$, respectively.
It is convenient to introduce a combination of the Laplace transform with respect to $t$ and a Fourier transform with respect to $x$ and $y$, for $z \leqslant 0$,

$$
\begin{aligned}
{\left[\bar{\eta}, \bar{\Phi}, \overline{\mathbf{V}}_{T}\right]=} & \int_{0}^{\infty} e^{-s t} \int_{-\infty}^{\infty} \int_{-\infty}^{\infty}\left[\eta, \Phi e^{-A z}, \mathbf{V}_{T} e^{-B z}\right] \\
& \times e^{-i \alpha x-i \beta y} \mathrm{~d} x \mathrm{~d} y \mathrm{~d} t
\end{aligned}
$$

where

$$
\begin{gathered}
A(\alpha, \beta)=\sqrt{\alpha^{2}+\beta^{2}}, \\
B(s, \alpha, \beta)=\sqrt{(s+i \alpha) / \varepsilon+\alpha^{2}+\beta^{2}} .
\end{gathered}
$$

Substituting Eqs. (14a)-(14c) into boundary conditions (10)-(13) and the continuity equation, we obtain a system of linear equations for the five unknown variables $\left(\bar{\eta}, \bar{\Phi}, \bar{u}_{T}, \bar{v}_{T}, \bar{w}_{T}\right)$, which can readily be solved. Upon some mathematical manipulation, the integral expression for the wave profile can be written as

$$
\begin{aligned}
\eta\left(x, y, t ; h_{0}\right)= & \frac{1}{8 \pi^{3} i} \int_{c-i \infty}^{c+i \infty} \int_{-\infty}^{\infty} \int_{-\infty}^{\infty} \frac{N}{D} \\
& \times \exp (i \alpha x+i \beta y+s t) \mathrm{d} \alpha \mathrm{d} \beta \mathrm{d} s,
\end{aligned}
$$

where

$$
\begin{aligned}
N= & -\left(s+i \alpha+2 \varepsilon A^{2}\right)\left(i \alpha F_{x}-A F_{z}\right) \exp \left(-A h_{0}\right) \\
& +2 \varepsilon A\left(i \alpha B F_{x}-A^{2} F_{z}\right) \exp \left(-B h_{0}\right), \\
D= & s(s+i \alpha)\left[A+\left(s+i \alpha+2 \varepsilon A^{2}\right)^{2}-4 \varepsilon^{2} A^{3} B\right],
\end{aligned}
$$

and $c$ is the convergence abscissa for the inverse Laplace transform.

The integral in Eq. (15a) represents the solution for the wave elevation due to a singular force, but the physical characteristics of the wave motion are not explicitly seen in this integral. Now the task is to extract information from Eq. (15a). As stated by Noblesse and Chen [32], the Fourier integral representation for wave profiles can formally be decomposed into a near-field nonoscillatory component and a far-field wave component. The near-field component will rapidly vanish as the distance from the singularity increases and will not be investigated in the present study while the far-field behavior for small $\varepsilon$ is of principal physical interest.

It is noted that for small $\varepsilon, D(s, \alpha, \beta)$ in Eq. (15c) has four simple poles with respect to $s$,

$$
\begin{gathered}
s_{j}=(-1)^{j+1} i \sqrt{A}-i \alpha-2 \varepsilon A^{2}+O\left(\varepsilon^{3 / 2}\right), \quad(j=1,2), \\
s_{3}=-i \alpha, \\
s_{4}=0 .
\end{gathered}
$$

The inversion of the Laplace transform in Eq. (15a) can be determined by the sum of the residues of the integrand at these poles. By taking a contour integration in the complex $s$ plane, Eq. (15a) can be represented by the double integral 


$$
\eta=\frac{1}{4 \pi^{2}} \sum_{j=1}^{4} \int_{-\infty}^{\infty} \int_{-\infty}^{\infty} \frac{N_{j}}{D_{0}} \exp [i(\alpha x+\beta y)] \mathrm{d} \alpha \mathrm{d} \beta,
$$

where

$$
\begin{aligned}
& N_{j}=\left.N\right|_{s=s_{j}}, \\
& D_{0}=\left.D\right|_{s=0} .
\end{aligned}
$$

To obtain the asymptotic expansion of the Fourier-type double integral in Eq. (17a), we shall employ Lighthill's twostage scheme [33], which in essence involves calculating the $\alpha$ integration by the residue theorem and the $\beta$ integration by the method of steepest descents. For small $\varepsilon, D_{0}(\alpha, \beta)$ in Eq. (17c) has two simple zeros with respect to $\alpha$,

$$
\alpha_{n}=(-1)^{n+1} a_{0}+i \varepsilon a_{1}+O\left(\varepsilon^{3 / 2}\right), \quad(n=1,2),
$$

where

$$
\begin{gathered}
a_{0}(\beta)=\left[1+\left(1+4 \beta^{2}\right)^{1 / 2}\right]^{1 / 2} / \sqrt{2}, \\
a_{1}(\beta)=4 a_{0}^{6}\left(2 a_{0}^{2}-1\right)^{-1} .
\end{gathered}
$$

We introduce the cylindrical coordinates $(R, \theta)$ on the horizontal $(x, y)$ plane by $x=R \cos \theta, y=R \sin \theta$. Using the residue theorem, the leading terms which contribute significantly to the wave profile in the far field can be written as

$$
\begin{aligned}
\eta= & \frac{1}{2 \pi} \sum_{j=0}^{4} \sum_{n=1}^{2} \int_{-\infty}^{\infty}\left\{\frac{a_{0}^{2} G_{j n} \exp \left[R f_{n}(\beta)\right]}{1-2 a_{0}^{2}}+o\left(\frac{1}{R}\right)\right\} \mathrm{d} \beta \\
& 0<R \cos \theta<t
\end{aligned}
$$

where

$$
\begin{gathered}
G_{j n}=\left.N_{j}\right|_{\alpha=\alpha_{n}}, \\
f_{n}(\beta)=\alpha_{n} \cos \theta+i \beta \sin \theta .
\end{gathered}
$$

For the second stage of Lighthill's scheme, the method of steepest descents should be employed for $\beta$ integration at large $R[14,15,19]$. The leading term of the solution for stationary points of the phase function is given by [31]

$$
\beta=\beta_{n m}=(-1)^{n} q_{m} Q_{m}^{1 / 2} \tan \theta, \quad(n, m=1,2),
$$

where

$$
\begin{gathered}
q_{m}=2\left[1+(-1)^{m+1} \sqrt{1-8 \tan ^{2} \theta}\right]^{-1}, \\
Q_{m}=\left(q_{m}+1\right) / 2 .
\end{gathered}
$$

By means of the steepest-descents analysis and other mathematical manipulations, the formal expression for the unsteady free-surface waves in the viscous far wake is given by

$$
\eta=H(t-R \cos \theta) \sum_{k=0}^{2}\left(\eta_{k}^{S}+\eta_{k}^{T}\right)+o\left(R^{-1}\right)
$$

$$
\eta_{0}^{S}=-d^{R} \sum_{m=1}^{2} d_{m}^{V} d_{m}^{S} Q_{m}^{3 / 4}\left[F_{x} \cos M_{m}-Q_{m}^{1 / 2} F_{z} \sin M_{m}\right]
$$

$$
\eta_{1}^{S}=-2 \varepsilon d^{R} \sum_{m=1}^{2} d_{m}^{V} d_{m}^{S} Q_{m}^{9 / 4}\left[F_{x} \sin M_{m}+Q_{m}^{1 / 2} F_{z} \cos M_{m}\right]
$$

$$
\begin{aligned}
\eta_{2}^{S}= & 2 \varepsilon^{1 / 2} d^{R} \sum_{m=1}^{2} d_{m}^{V} d_{m}^{B} Q_{m}^{3 / 2}\left[F_{x} \sin \left(M_{m}-\frac{Q_{m}^{1 / 4} h_{0}}{\sqrt{2 \varepsilon}}+\pi / 4\right)\right. \\
& +\varepsilon^{1 / 2} F_{z} Q_{m}^{5 / 4} \cos \left(M_{m}-\frac{Q_{m}^{1 / 4} h_{0}}{\sqrt{2 \varepsilon}}\right)
\end{aligned}
$$

$$
\eta_{0}^{T}=-d^{R} \sum_{m=1}^{2} d_{m}^{V} d_{m}^{S} d_{m}^{T} Q_{m}^{3 / 4}\left[F_{x} \cos M_{m}-Q_{m}^{1 / 2} F_{z} \sin M_{m}\right]
$$

$$
\begin{aligned}
& \eta_{1}^{T}=-\varepsilon d^{R} \sum_{m=1}^{2} d_{m}^{V} d_{m}^{S} d_{m}^{T} Q_{m}^{9 / 4}\left[F_{x}\left(3 \sin M_{m}-\sin N_{m}\right)\right. \\
&\left.+Q_{m}^{1 / 2} F_{z}\left(3 \cos M_{m}-\cos N_{m}\right)\right], \\
& \eta_{2}^{T}=2 \varepsilon^{1 / 2} d^{R} \sum_{m=1}^{2} d_{m}^{V} d_{m}^{B} d_{m}^{T} Q_{m}^{3 / 2}\left[F_{x} \sin \left(M_{m}-\frac{Q_{m}^{1 / 4} h_{0}}{\sqrt{2 \varepsilon}}+\pi / 4\right)\right. \\
&\left.+\varepsilon^{1 / 2} F_{z} Q_{m}^{5 / 4} \cos \left(M_{m}-\frac{Q_{m}^{1 / 4} h_{0}}{\sqrt{2 \varepsilon}}\right)\right]
\end{aligned}
$$

and

$$
\begin{gathered}
d^{R}=\left(\frac{2}{\pi R \cos \theta}\right)^{1 / 2}\left(1-8 \tan ^{2} \theta\right)^{-1 / 4}, \\
d_{m}^{V}=\exp \left(-4 \varepsilon q_{m}^{-1} Q_{m}^{3} R \cos \theta\right) \\
d_{m}^{S}=\exp \left(-Q_{m} h_{0}\right) \\
d_{m}^{B}=\exp \left(-\frac{Q_{m}^{1 / 4} h_{0}}{\sqrt{2 \varepsilon}}\right) \\
d_{m}^{T}=\exp \left(-2 \varepsilon Q_{m}^{2} t\right)
\end{gathered}
$$

$$
M_{m}=Q_{m}^{1 / 2} R \cos \theta-q_{m} Q_{m}^{1 / 2} R \sin \theta \tan \theta+(-1)^{m+1} \frac{\pi}{4},
$$

$$
N_{m}=Q_{m}^{1 / 2} R \cos \theta-q_{m} Q_{m}^{1 / 2} R \sin \theta \tan \theta+(-1)^{m+1} \frac{\pi}{4}-2 Q_{m}^{1 / 2} t
$$




\section{UNSTEADY VISCOUS FREE-SURFACE WAVES DUE TO A SUDDENLY STOPPING OSEENLET}

In Sec. III, the point force is assumed to start suddenly from rest and to move with a constant velocity. In this section, the point force is assumed to stop suddenly from a steady state, the decaying of three-dimensional waves will be investigated. Cartesian coordinates are taken fixed on the singularity. It is assumed that the point force stops suddenly at $t=0$. For $t<0$ the steady-state flow is due to an Oseenlet.

The governing equations are Eqs. (1) and (2) with $\mathbf{F}_{S}=0$. The boundary conditions are given by Eqs. (3)-(6). We may write $\mathbf{u}(x, z, t)=\nabla \Phi+\mathbf{V}_{T}$, where $\Phi(\mathbf{x}, t)$ represents an irrotational flow while $\mathbf{V}_{T}(\mathbf{x}, t)$ represents a rotational flow. Thus, the boundary conditions can be expressed by Eqs. (10)-(13) with $u_{S}=v_{S}=w_{S}=P_{S}=0$. The initial conditions can be given by $\left.\eta(x, y, t)\right|_{t=0}=\eta^{S}(x, y)$ and $\left.\Phi(x, y, 0, t)\right|_{t=0}=\Phi^{S}(x, y, 0)$, where $\eta^{S}$ is the steady free-surface wave elevation, and $\Phi^{S}$ is the velocity potential. Following Sec. III, one may obtain the steady wave elevation and the potential function as

$$
\begin{aligned}
\left\{\eta^{S}(x, y), \Phi^{S}(x, y, 0)\right\}= & \frac{1}{4 \pi^{2}} \int_{-\infty}^{\infty} \int_{-\infty}^{\infty}\left\{N_{\eta}^{S}, N_{\Phi}^{S}\right\} \\
& \times \frac{\exp (i \alpha x+i \beta y)}{i \alpha D_{0}} \mathrm{~d} \alpha \mathrm{d} \beta,
\end{aligned}
$$

where

$$
\begin{gathered}
N_{\Phi}^{S}=\left(i \alpha F_{x}-A F_{z}\right)\left[\frac{1}{2 A}\left(\alpha^{2}+A\right)-2 i \varepsilon \alpha A-2 \varepsilon^{2} A^{2}\left(A+B_{0}\right)\right] \\
\times \exp \left(-A h_{0}\right)+2\left(B_{0} \alpha F_{x}+i A^{2} F_{z}\right)\left(\alpha \varepsilon-2 i A^{2} \varepsilon^{2}\right) \\
\times \exp \left(-B_{0} h_{0}\right), \\
\qquad N_{\eta}^{S}=\left.N\right|_{s=0}, \\
B_{0}=\left.B\right|_{s=0} .
\end{gathered}
$$

The procedure to obtain $\eta(x, y, t)$ follows that in Sec. III and will not be reproduced here. Finally, the unsteady freesurface waves in the viscous far wake become

$$
\eta=\sum_{k=0}^{4} \eta_{k}^{T}+o\left(\frac{1}{R}\right)
$$

where

$$
\eta_{1}^{T}=-2 \varepsilon d^{R} \sum_{m=1}^{2} d_{m}^{V} d_{m}^{S} d_{m}^{T} Q_{m}^{9 / 4}\left[F_{x} \sin M_{m}+Q_{m}^{1 / 2} F_{z} \cos M_{m}\right]
$$

$$
\begin{aligned}
\eta_{2}^{T}= & -2 \varepsilon^{3 / 2} d^{R} \sum_{m=1}^{2} d_{m}^{V} d_{m}^{S} d_{m}^{T} Q_{m}^{3} \\
& \times\left\{F_{x}\left[\cos \left(M_{m}+\frac{\pi}{4}\right)-\cos \left(N_{m}+\frac{\pi}{4}\right)\right]\right. \\
& \left.-Q_{m}^{1 / 2} F_{z}\left[\sin \left(M_{m}+\frac{\pi}{4}\right)-\sin \left(N_{m}+\frac{\pi}{4}\right)\right]\right\},
\end{aligned}
$$

$$
\begin{aligned}
\eta_{3}^{T}= & \varepsilon^{3 / 2} d^{R} \sum_{m=1}^{2} d_{m}^{V} d_{m}^{S} d_{m}^{T} Q_{m}^{15 / 4}\left[F_{x}\left(\cos M_{m}-\cos N_{m}\right)\right. \\
& \left.-Q_{m}^{1 / 2} F_{z}\left(\sin M_{m}-\sin N_{m}\right)\right], \\
\eta_{4}^{T}= & 2 \varepsilon^{1 / 2} d^{R} \sum_{m=1}^{2} d_{m}^{V} d_{m}^{B} d_{m}^{T} Q_{m}^{3 / 2}\left[F_{x} \sin \left(M_{m}-\frac{Q_{m}^{1 / 4} h_{0}}{\sqrt{2 \varepsilon}}+\frac{\pi}{4}\right)\right. \\
& \left.-\varepsilon^{1 / 2} F_{z} Q_{m}^{5 / 4} \cos \left(M_{m}-\frac{Q_{m}^{1 / 4} h_{0}}{\sqrt{2 \varepsilon}}\right)\right],
\end{aligned}
$$

where $\eta_{0}^{T}, Q_{m}^{1 / 4}, d^{R}, d_{m}^{V}, d_{m}^{S}, d_{m}^{B}, d_{m}^{T}, M_{m}$, and $N_{m}$ are given in Sec. III.

\section{CHARACTERISTICS OF UNSTEADY VISCOUS WAVES DUE TO AN OSEENLET}

It can be seen from Eq. (21) that the disturbed waves consist of two parts, $\eta_{k}^{S}$ and $\eta_{k}^{T}$. As time tends to infinity, $\eta_{k}^{S}$ reduces to the steady viscous waves and $\eta_{k}^{T}$ vanishes due to the exponential temporal decay factor $d_{m}^{T}$. Therefore, $\eta_{k}^{S}$ is the steady-state response and $\eta_{k}^{T}$ the transient response to an Oseenlet starting suddenly from rest to a constant velocity. It is noted that the expressions of wave profiles involving $q_{1}$ correspond to the transverse wave system, while the expressions involving $q_{2}$ the diverging wave system. Accordingly, the solution for the unsteady waves is given by the superposition of four families of waves: the steady transverse waves, the steady diverging waves, the transient transverse waves, and the transient diverging waves.

It should be noted that the amplitudes of $\eta_{1}^{S}$ and $\eta_{1}^{T}$ are of order $\varepsilon$ relative to those of $\eta_{0}^{S}$ and $\eta_{0}^{T}$, whereas $\eta_{2}^{S}$ and $\eta_{2}^{T}$ are trivial components for large submergence depths due to the presence of factor $d_{m}^{B}$. $\eta_{2}^{S}$ and $\eta_{2}^{T}$ become relatively significant only when $h_{0}=O(\sqrt{\varepsilon})$, that is, when the Oseenlet is near the free surface. Thus, $\eta_{k}^{S}$ and $\eta_{k}^{T}(k=1,2)$ can be regarded as the higher-order viscous correction to the wave profile. In addition, there exists a phase shift of $\pi / 2$ between $\eta_{0}^{S}$ and $\eta_{1}^{S}$, which is also true for $\eta_{0}^{T}$ and $\eta_{1}^{T}$. The phases of $\eta_{2}^{S}$ and $\eta_{2}^{T}$ are shifted due to the presence of the submergence depth. An interesting feature of the present result is that the first-order solution for the transient response, $\eta_{1}^{T}$, consists of two waves with different phases of oscillation, i.e., $M_{m}$ and $N_{m}$, which represent the "noise" disturbance in the initial stage of the wave evolution and decay exponentially as time increases. Although the contribution from the first-order solution remains insignificant, it represents the effect of a viscous wake.

Equation (21) shows that the generated unsteady viscous waves are confined within a wedge of semiangle as well as within a moving finite triangular region behind the body,

$$
\Lambda_{\eta}=\left\{(R, \theta)|0<R \leqslant t \sec \theta,| \theta \mid<\theta_{c}\right\},
$$

where $\theta_{c}=\tan ^{-1} \sqrt{1 / 8} \approx 19^{\circ} 28^{\prime}$. The moving finite regions are shown in Fig. 2, which is consistent with that obtained by Liu and Tao [6] for the unsteady ship waves in an inviscid fluid. As time increases, the waves move away from the body and the finite triangular region extends to an infinite $\mathrm{V}$-shaped region, which is the same as the well-known Kelvin ship-wave wedge for inviscid waves. 


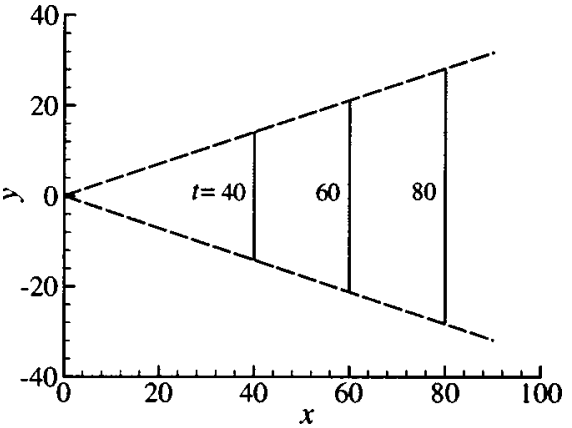

FIG. 2. The moving triangular region for unsteady free-surface waves at $t=40,60$, and 80 ( - , boundary line; ---, cusp line).

Dugan [12] found that in two-dimensional Oseen flows, the wave amplitude due to a unity drag is the same as that due to a unit lift while there is a phase shift of $\pi / 2$ between these two waves. Equation (21) shows that for the threedimensional case, the wave amplitude due to a unity drag is less than that due to a unit lift since $Q_{m} \geqslant 1$, and there also exists a phase shift of $\pi / 2$ between these two waves, as shown in Fig. 3.

A close examination of the solutions shows that there exist three types of decay factors for the steady wave profiles: the radial decay factor $d^{R}$, the viscous decay factor $d_{m}^{V}$, and the submergence decay factors $d_{m}^{S}$ and $d_{m}^{B}$. All generated waves are attenuated algebraically along the distance away from the body as well as exponentially by the inclusion of viscosity and submergence. The effects of parameters $\varepsilon$ and $h_{0}$ on the wave profile are shown in Figs. 4 and 5, respectively. Besides these three factors, there is a temporal decay factor $d_{m}^{T}$ for transient waves, which ensures that the transient components eventually vanish as time goes to infinity. Figure 6 shows the unsteady viscous waves at different instants. As time increases, the transient waves diminish. Thus, the steady state $(t \rightarrow \infty)$ can be attained ultimately.

A careful examination of factor $d_{m}^{T}$ for the transverse $(m$ $=1)$ and diverging waves $(m=2)$ shows that the transverse wave system is less damped than the diverging one, especially in the region close to $\theta=0$. Therefore, for a point singularity, the transverse wave system is the dominant component of the singularity-generated wave system.

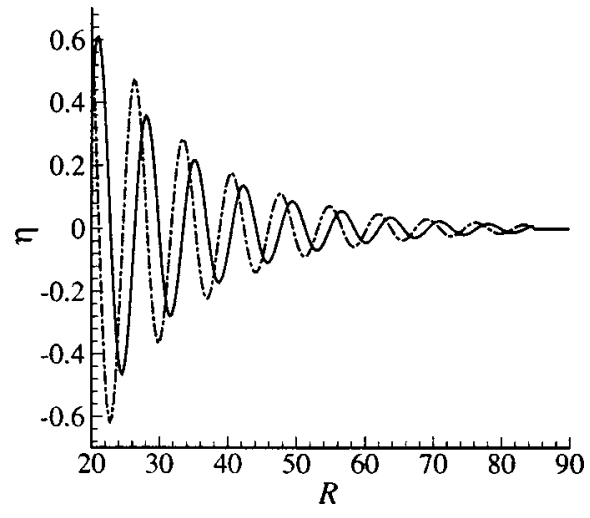

FIG. 3. A comparison between drag- and lift-induced waves with $h_{0}=1, \varepsilon=0.01, F_{x}=4 \pi, F_{z}=4 \pi, \theta=0.33$, and $t=80$ (一, draginduced wave; ----., lift-induced wave).

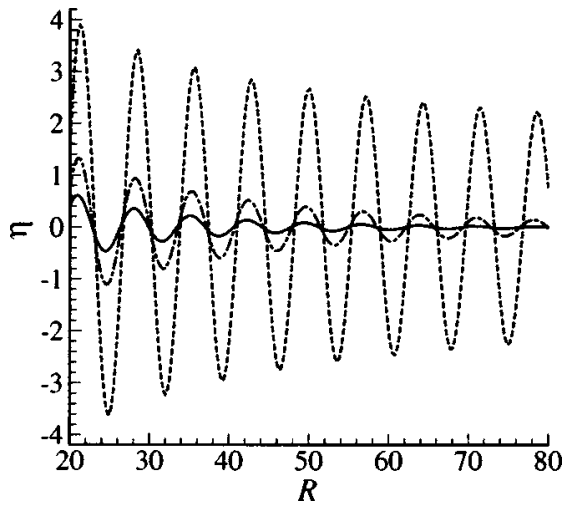

FIG. 4. The effect of parameter $\varepsilon$ on the wave profiles with $h_{0}$ $=1, \varepsilon=0.01, F_{x}=4 \pi, F_{z}=0, \theta=0.33$, and $t=80$ (---, $\varepsilon=0 ;---\cdot, \varepsilon$ $=0.005 ;-, \varepsilon=0.01)$.

A special case of the present result with $\varepsilon=0$ and $F_{z}=0$ corresponds to a simple source moving in an inviscid fluid. In the limit as $\varepsilon$ tends to zero, $\eta_{1}^{S}, \eta_{2}^{S}, \eta_{1}^{T}$, and $\eta_{2}^{T}$ vanish while $\eta_{0}^{S}$ and $\eta_{0}^{T}$ simply reduce to the same one. Thus, the steady and transient components of the surface elevation for an inviscid fluid are

$$
\eta_{S, 0}=\eta_{T, 0}=-F_{x} d^{R} \sum_{m=1}^{2} d_{m}^{S} Q_{m}^{3 / 4} \cos M_{m}+o\left(R^{-1}\right), \quad 0<x<t
$$

which agrees with that obtained by Liu and Tao $[[6]$, Eq. (35)]. The remarkable difference between the present solution and the corresponding inviscid solution predicted by Liu and Tao [6] is the existence of a temporal decay factor $d_{m}^{T}$. It can be seen from Eq. (38) that the potential theory predicts a nonvanishing transient component. This incompatibility caused by the potential theory is removed by the viscous theory.

A special case of the present result with $F_{z}=0$ corresponds to a "singular needle" moving in a viscous flow. The corresponding solution obtained by Lurye [[14], Eqs. (69) and (70)] is recovered by the present solution. Another special case of the present result with $F_{x}=0$ and $h_{0}=0$ corresponds

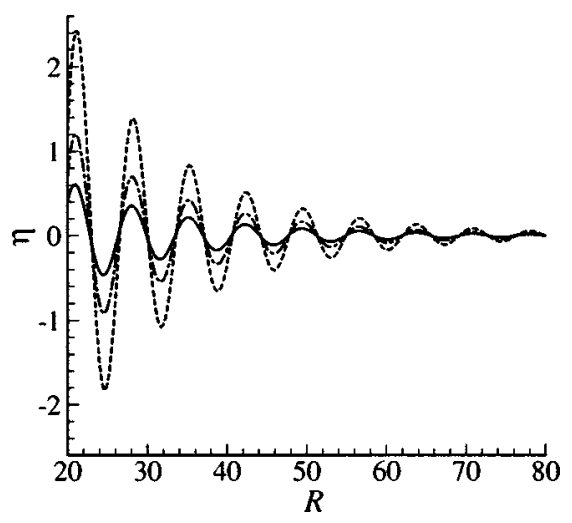

FIG. 5. The effect of parameter $h_{0}$ on the wave profiles with $h_{0}=1, \varepsilon=0.01, F_{x}=4 \pi, F_{z}=0, \theta=0.33$, and $t=80\left(---, h_{0}=0 ;-\cdot-\cdot\right.$, $\left.h_{0}=0.5 ;-, h_{0}=1.0\right)$. 


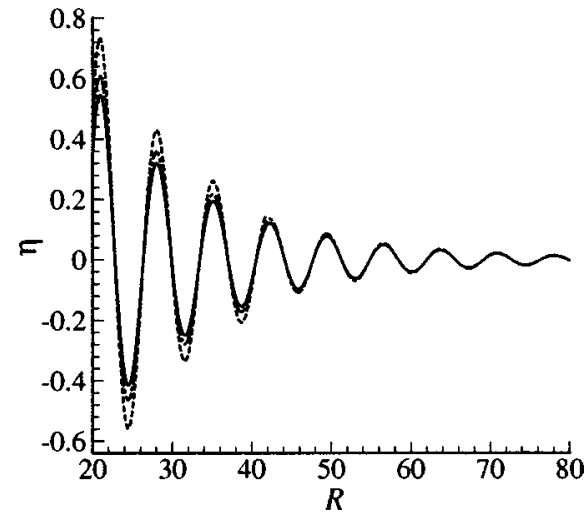

FIG. 6. The evolution of viscous free-surface waves with $h_{0}$ $=1, \varepsilon=0.01, F_{x}=4 \pi, F_{z}=0$, and $\theta=0.33$ (---, $t=40 ;---\cdot, t=80 ;-$ $t=\infty)$.

to a point pressure moving on the surface of a viscous fluid. The corresponding steady solution given by Cumberbatch [[9], Eq. (48)] is also recovered by the present solution. It should be noted that the potential theories predict an infinite amplitude for diverging waves near the moving path of a floating body [[5], Fig. 4]. However, the viscous theory presented in this paper predicts zero amplitude for diverging waves as $\theta$ approaches the path of a moving body, which is consistent with the physical reality.

In the limit as $\varepsilon$ tends to zero, Eq. (32) reduces to the solution obtained by Wehausen and Laitone [4] for steady inviscid waves. The most remarkable feature of Eq. (32) is the presence of viscous decay factors and high-order viscous corrections. $d_{m}^{T}(m=1,2)$ can be regarded as decay factors for the energy dissipation in ship waves. From Eqs. (32)-(36) and Fig. 7, it can easily be seen that the wave will die out as time goes to infinity. However, the potential theory predicts a nonvanishing wave for the suddenly stopping singularity, which does not agree with the physical observation. It can be stated that the attenuation of waves is caused by the inclusion of viscosity.

\section{CONCLUSIONS}

Analytical solutions are obtained for the unsteady viscous free-surface waves generated by a point force moving in an incompressible fluid of infinite depth. The point force is assumed to experience a Heaviside step change in velocity at the initial instant. Two categories of the velocity change, (i) from zero to a constant and (ii) from a constant to zero, have been analyzed. It is found that the unsteady viscous wave

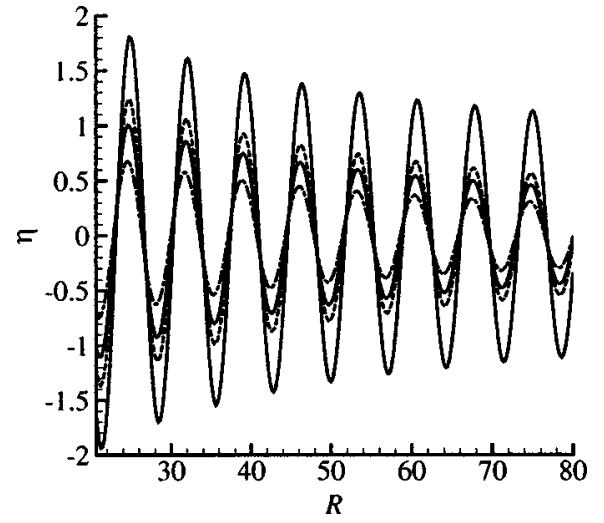

FIG. 7. The evolution of inviscid waves $(-\cdot \cdot-)$ and of viscous waves with $\varepsilon=0.001, h_{0}=1, F_{x}=4 \pi$, and $\theta=0.33$. (----- $t=50$, $t=100,-\cdot-\cdot-t=200$ for viscous waves).

system consists of four families of waves: the steady transverse waves, the steady diverging waves, the transient transverse waves, and the transient diverging waves. The generated unsteady waves are confined within a finite triangular region and move away behind the point force. As time increases, the finite region extends to an infinite V-shaped region. The waves due to a suddenly stopping Oseenlet consist of two families of waves: the transient transverse waves and the transient diverging waves.

All waves are attenuated algebraically along the distance away from the Oseenlet as well as exponentially by the inclusion of viscosity and submergence. The remarkable feature for the transient viscous waves is the existence of a temporal decay factor, which ensures that the transient components will tend to zero as time goes to infinity. Thus, the steady state can be attained ultimately. The radial decay factor, the submergence decay factors, and the viscous decay factors (one spatial and another temporal) are analytically expressed. The diverging waves are more severely damped than the transverse waves. Hence the singularity-induced wave system consists primarily of transverse waves.

It is found that the fundamental singularity in Oseen flow (Oseenlet) is a general model for the simulation of a moving body on or beneath the free surface of a real fluid since the previous works by other authors can readily be attained by taking the corresponding limits of the present result.

\section{ACKNOWLEDGMENTS}

This research was sponsored by the Hong Kong Research Grants Council under Grant No. HKU 7076/02E and the Youth Foundation of Shanghai Municipal Education Commission under Grant No. 04AC79.
[1] A. T. Chwang and T. Y. Wu, J. Fluid Mech. 67, 787 (1975).

[2] F. H. Clauser, Phys. Fluids 26, 2787 (1983).

[3] T. Dabros, J. Fluid Mech. 156, 1 (1985).

[4] J. V. Wehausen and E. V. Laitone, in Encyclopedia of Physics IX: Fluid Dynamics III, edited by S. Flügge (Springer-Verlag, Berlin, 1960), pp. 483-493.
[5] Y. K. Chung and J. S. Lim, J. Ship Res. 35, 191 (1991).

[6] M. J. Liu and M. D. Tao, Phys. Fluids 13, 3610 (2001).

[7] D. Q. Lu and A. T. Chwang, in Proceedings of the 2nd International Conference on Asian and Pacific Coasts (CD-ROM), Makuhari Messe, Chiba, Japan, 2004, edited by Y. Goda, W. Kioka, and K. Nadaoka, APAC021, pp. 1-9. 
[8] T. Y. Wu and R. R. Messick, Caltech. Report No. 85-8 (1958).

[9] E. Cumberbatch, J. Fluid Mech. 23, 471 (1965).

[10] J. R. Lurye, Phys. Fluids 10, 261 (1968).

[11] R. Brard, J. Ship Res. 14, 207 (1970).

[12] J. P. Dugan, Phys. Fluids 12, 1 (1969).

[13] E. Ammicht, J. Eng. Math. 13, 327 (1979).

[14] J. R. Lurye, Phys. Fluids 16, 750 (1973).

[15] A. T. Chan and A. T. Chwang, Phys. Fluids 8, 421 (1996).

[16] A. T. Chan and A. T. Chwang, Phys. Fluids 9, 940 (1997).

[17] M. J. Liu and M. D. Tao, Appl. Math. Mech. 23, 1221 (2002).

[18] D. Q. Lu, J. Hydrodynam. 15(4), 10 (2003).

[19] D. Q. Lu, Appl. Math. Mech. 25, 647 (2004).

[20] D. Q. Lu, J. Hydrodynam. 17, 22 (2005).

[21] P. A. Tyvand, Free Surface Flows With Viscosity (Computational Mechanics Publications, Southampton, UK, 1998), p. ix.

[22] M. R. Maxey and J. J. Riley, Phys. Fluids 26, 883 (1983).

[23] C. Pozrikidis, Phys. Fluids A 1, 1508 (1989).

[24] W. G. Price and M. Y. Tan, Proc. R. Soc. London, Ser. A 438, 447 (1992).
[25] A. T. Chan and A. T. Chwang, Proc. Inst. Mech. Eng., Part C: J. Mech. Eng. Sci. 214, 175 (2000).

[26] J. J. Shu and A. T. Chwang, Phys. Rev. E 63, 051201 (2001).

[27] J. J. Shu and A. T. Chwang, in Proceedings of the 8th Asian Congress of Fluid Mechanics, Shenzhen, China (International Academic Publishers, Beijing, China, 1999), p. 321.

[28] J. J. Shu and A. T. Chwang, in Proceedings of the 14th Eng. Mech. Conference, Austin, USA (CD-ROM), (2000), 6 pp.

[29] D. Q. Lu and A. T. Chwang, in Proceedings of the 1st Asian and Pacific Coastal Engineering Conference, Dalian, China, 2001, edited by D. H. Qiu and Y. C. Li (Dalian University of Technology Press, Dalian, China, 2001), p. 334.

[30] D. Q. Lu and A. T. Chwang, J. Ship Mech. 8, 8 (2004).

[31] D. Q. Lu, Ph.D. thesis, The University of Hong Kong, 2002.

[32] F. Noblesse and X. B. Chen, Ship Technology Res. 42, 167 (1995).

[33] M. J. Lighthill, Philos. Trans. R. Soc. London, Ser. A 252, 397 (1960). 